\title{
Handwritten Digit Recognition Based on Support Vector Machine
}

\author{
Xinwen Gao ${ }^{a}$, Benbo Guan ${ }^{b}$ and Liqing $Y u^{c}$ \\ School of Mechatronic Engineering and Automation, Shanghai University, Shanghai 200072, China \\ agxw3405@shu.edu.cn, bguanbenbo@163.com, c $429346220 @ q q . c o m$
}

Keywords: Handwriting recognition, Support Vector Machine, Feature extraction, Normalization, Kernel function.

\begin{abstract}
In this paper, we propose a handwritten digit recognition method based on Support Vector Machined (SVM). Firstly, some main features are extracted from the handwritten digital images (Euler Number, roundness, moment feature, crossing density, pixel density). Secondly, adopt the method of SVM for classification. This paper adds the feature values normalized, using radial basis function and Cross-validation important parameters. Our approach has been implemented with MNIST database and we have achieved an average recognition rate of $96.3 \%$, the lowest single digit recognition rate of $93.5 \%$ when the training data are $100 \times 10$.
\end{abstract}

\section{Introduction}

Handwritten digit recognition is a branch of optical character recognition technology. The main research of the handwritten digit recognition is how to use the computer automatically recognize handwritten digits. Due to the extensive use of digital symbols (such as postal code, bank check, financial forms), the Handwritten digit recognition technology is widely attention. It has been one of the hotspots with high practical value in the field of pattern recognition.

At present there are four main methods in the field of handwritten digit recognition. They are Bayesian Classification [1], Clustering Analysis [2], Neural Network [3] and SVM [4, 5]. A major problem in handwriting recognition is the huge variability and distortions of patterns. Elastic models based on local observations and dynamic programming such Hidden Markov Model (HMM) is not efficient to absorb this variability [6].The SVM is a machine learning algorithm developed based on the statistical theory, and it has advantages in solving small sample size and nonlinear pattern recognition problems.

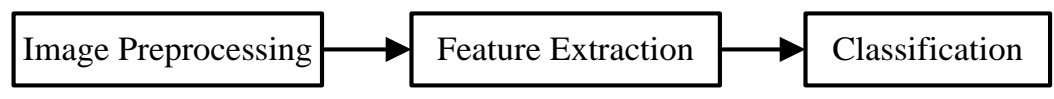

Fig. 1 Three main operations

As shown in Fig. 1, there are three main parts of handwritten digit recognition (image preprocessing, feature extraction and classification). Different features have different impact on the recognition rate, the feature extraction plays a key role in the recognition. Some scholars focus on the study of the feature extraction [7, 8]. In this paper, we select the SVM as a classification method. In the next part, we will describe the selected features in detail. Meanwhile, in order achieve higher recognition rate we adopt some optimization steps before the data training operation.

\section{Feature Extraction}

In this section, several main features used by this paper will be introduced.

Euler Number. Euler number is a topological parameters used to describe the object structure. Euler number is defined as: the difference between the connecting number and the number of holes. It can be expressed in the following formula:

$E=L-K$

$E$ represents the value of the Euler number, $L$ represents the number of the connecting, $K$ represents the number of holes. 
Roundness. Roundness feature can be used to describe the complexity of the object boundary. Roundness obtained by the ratio of the circumference and area of the square. It can be expressed in the following formula:

$$
A=\frac{C^{2}}{4 \pi S}
$$

$C$ is the circumference, $S$ is the area of the region.

Moment Feature. Hu proposed seven invariant moments, and he proves these moment features translation invariance and scale invariance and rotation invariance properties [9]. Considering the efficiency of calculation, we only extract the first two features. The formula as follows:

$$
\begin{aligned}
& \phi_{1}=\mu_{20}+\mu_{02} \\
& \phi_{2}=\left(\mu_{20}-\mu_{02}\right)^{2}+4 \mu_{11}^{2}
\end{aligned}
$$

Crossing Density. The crossing density is widely used in the study of digital identification. The basic idea is to use one or several groups of parallel lines throughout the digit, extracting the digit with parallel intersection information. This method involves digit topology information, has high tolerance for digital shape change. So, it is suitable for recognition the handwritten digits.

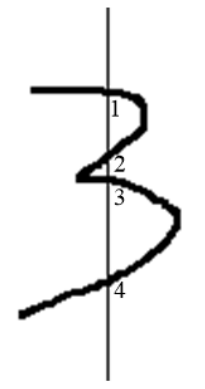

Fig. 2 crossing density feature

As shown in Fig.1, a vertical line through the number 3 and get four intersections. In the article, we extract the horizontal maximum number of intersections $M a x H$, average number $A v g H$. vertical direction maximum number of intersections $M a x V$, average number $A v g V$.

Pixel Density. The pixel density feature represents the different digits have the different number of black pixels within the same height at the top and bottom.

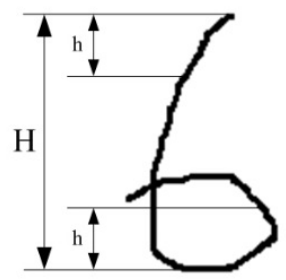

Fig. 3 Pixel density feature

As shown in Fig.2, in the same h distance range of number 6 from the top and bottom, the number of pixels at the bottom more than the top. In general, we get the $h \leqslant 0.2 H$. Record number of bottom pixels as DNum, the number of top pixels as UNum.

\section{Experiment and Result}

Based on the foregoing, we get ten features. Euler number $(E)$, Roundness feature $(A)$, Moment Feature $\left(\phi_{1}, \phi_{1}\right)$, Crossing density feature (MaxH, AvgH, MaxV, AvgV), Pixel Density feature (DNum, UNum). We will according to these features achieve the recognition of handwritten digits with SVM. The test digits are from MNIST database. We do the experiment with Chih-Jen Lin's LIBSVM package [10]. The experiment steps are as follows:

Step1 Normalized the features' values. In order to reduce the influence of different feature's values on the recognition results, we normalize the features' values. The formula as follows: 


$$
X^{*}=\frac{X=\operatorname{Min} X}{\operatorname{Max} X-\operatorname{Min} X}
$$

$\operatorname{Max} X$ is the maximum value of the same feature data, $\operatorname{Min} X$ is the minimum value of the same feature data. $X^{*} \in[0,1]$.

Step2 Kernel function. It is needed to map the features of low dimensional space to high dimensional space because of the classification boundaries of the handwritten digits recognition are nonlinear. In this paper, the radial basis function is selected. The formula as follows:

$$
K_{(x, z)}=\exp \left(-g\|x-z\|^{2}\right), g>0
$$

Step3 Determine the best value of $C$ and $g . C$ is the penalty parameter, $g$ is the span coefficient of radial kernel function. In general, the smaller value of $\mathrm{C}$, the higher fault tolerance. In this paper, using the cross-validation method to obtain the best $(C, g)$.After tests, the best value $(1.5157,0.05)$.

Table 1 the Experimental Results

\begin{tabular}{c|c|c|c}
\hline \multirow{2}{*}{ Digital } & \multirow{2}{*}{ Training Data } & \multicolumn{2}{|c}{ Prediction } \\
\cline { 3 - 4 } & & Image Data & Correct Rate \\
\hline 0 & 100 & 200 & $97 \%$ \\
\hline 1 & 100 & 200 & $95.5 \%$ \\
\hline 2 & 100 & 200 & $98.5 \%$ \\
\hline 3 & 100 & 200 & $98 \%$ \\
\hline 4 & 100 & 200 & $98.5 \%$ \\
\hline 5 & 100 & 200 & $96 \%$ \\
\hline 6 & 100 & 200 & $97 \%$ \\
\hline 7 & 100 & 200 & $94 \%$ \\
\hline 8 & 100 & 200 & $93.5 \%$ \\
\hline 9 & 100 & 200 & $95 \%$ \\
\hline Average & 100 & 200 & $96.3 \%$ \\
\hline
\end{tabular}

From the above Table 1 , we can learn that the training data are $100 \times 10$, the testing data are 200 $\times 10$. The proposed method has reached an average recognition rate of $96.3 \%$, and each digit recognition rate above $93 \%$. The experimental results show that our method is effective.

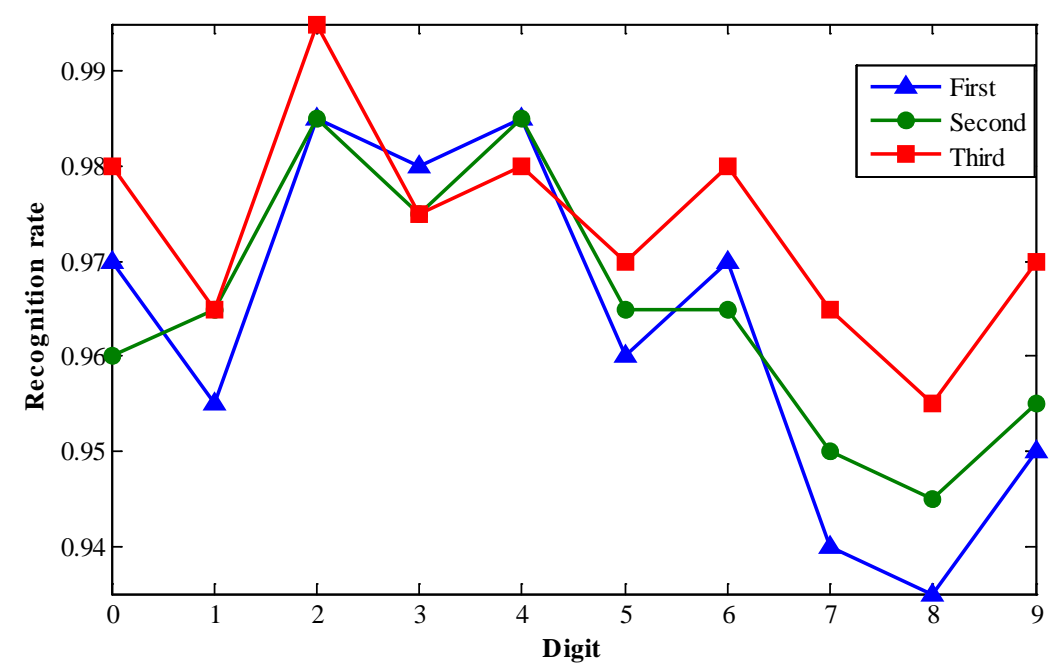

Fig. 4 crossing density feature

Finally, we made a comparison test on the different numbers of training data. The first group training data are $100 \times 10$, the second group training data are $200 \times 10$, the third group training data are $300 \times 10$. The Fig. 4 shows the more training data with the higher recognition rate. But the improvement is not obvious. We should pay more attention to features extraction to gain the higher recognition rate. 


\section{Conclusions}

This paper proposes a handwritten digit recognition method based on SVM. We extracted the ten main features and optimized the characteristic parameter values. The experimental result shows that this approach has reached an average recognition rate of $96.3 \%$.In the future, we will continue to do further research. Our goal is to increase the average recognition rate to $99 \%$, and the lowest single digital recognition rate is over $98 \%$. At the same time, we are considering to add handwritten English letters to the recognition system, expand the range of application.

\section{References}

[1] O. Pauplin, J. Jiang, ”A Dynamic Bayesian Network Based Structural Learning towards Automated Handwritten Digit Recognition”, Proc. of HAIS the 5th international conference on Hybrid Artificial Intelligence Systems, 2010, pp.120-127.

[2] Impedovo S, Mangini F M. A Novel Technique For Handwritten Digit Classification using Genetic Clustering[C]//Proceedings of the 2012 International Conference on Frontiers in Handwriting Recognition. IEEE Computer Society, 2012: 236-240.

[3] Hai T S, Thuy N T. Image Classification using Support Vector Machine and Artificial Neural Network [J]. International Journal of Information Technology and Computer Science (IJITCS), 2012, 4(5): 32.

[4] Neves R F P, Mello C A B, Zanchettin C. A SVM based off-line handwritten digit recognizer[C]//Systems, Man, and Cybernetics (SMC), 2011 IEEE International Conference on. IEEE, 2011: 510-515.

[5] Neves R F P, Zanchettin C, Lopes Filho A N G. An efficient way of combining SVMs for handwritten digit recognition [M]//Artificial Neural Networks and Machine Learning-ICANN 2012. Springer Berlin Heidelberg, 2012: 229-237.

[6] Sharma A. Handwritten digit Recognition using Support Vector Machine [J]. arXiv preprint arXiv:1203.3847, 2012.

[7] De Stefano C, Fontanella F, Marrocco C, et al. A GA-based feature selection approach with an application to handwritten character recognition [J]. Pattern Recognition Letters, 2014, 35: 130-141.

[8] Nguyen M H, De la Torre F. Optimal feature selection for support vector machines [J]. Pattern recognition, 2010, 43(3): 584-591.

[9] $\mathrm{Hu} \mathrm{M} \mathrm{K}$. Visual pattern recognition by moment invariants [J]. Information Theory, IRE Transactions on, 1962, 8(2): 179-187.

[10] Information on http://www.csie.ntu.edu.tw/ cjlin/ 